\title{
WekaDeeplearning4j: a Deep Learning Package for Weka based on DeepLearning4j
}

\author{
Steven Lang ${ }^{\mathrm{a}}$, Felipe Bravo-Marquez ${ }^{\mathrm{b}}$, Christopher Beckham ${ }^{\mathrm{c}}$, Mark Hall ${ }^{\mathrm{d}}$, \\ Eibe Frank ${ }^{\mathrm{e}}$ \\ ${ }^{a}$ Technische Universität Darmstadt, Darmstadt Germany \\ ${ }^{b}$ Department of Computer Science, University of Chile $\mathcal{E}$ IMFD, Santiago, Chile \\ ${ }^{c}$ École Polytechnique de Montréal, Montréal, Canada \\ ${ }^{d}$ Hitachi Vantara \\ ${ }^{e}$ Department of Computer Science, University of Waikato, Hamilton, New Zealand
}

\begin{abstract}
Deep learning is a branch of machine learning that generates multi-layered representations of data, commonly using artificial neural networks, and has improved the state-of-the-art in various machine learning tasks (e.g., image classification, object detection, speech recognition, and document classification). However, most popular deep learning frameworks such as TensorFlow and PyTorch require users to write code to apply deep learning. We present WekaDeeplearning4j, a Weka package that makes deep learning accessible through a graphical user interface (GUI). The package uses Deeplearning4j as its backend, provides GPU support, and enables GUI-based training of deep neural networks such as convolutional and recurrent neural networks. It also provides pre-processing functionality for image and text data.
\end{abstract}

Keywords: Deep Learning, Weka

\section{Introduction}

We present WekaDeeplearning4 1 , a tool for training and testing deep learning models implemented in Deeplearning4.j2 from within Weka [1], a widely used open-source machine learning workbench implemented in Java.

${ }^{1}$ https://deeplearning.cms.waikato.ac.nz/ 
The main goal of our tool is to make deep learning accessible without requiring users to write code - via Weka's GUI. At the most basic level, the GUI enables users to perform experiments using the following simple steps: 1) loading data in the Attribute-Relation File Format (ARFF) $\left.{ }^{3}, 2\right)$ configuring a neural network architecture, 3) choosing an experimental protocol, and 4) running the experiment.

The WekaDeeplearning4j package supports fully connected feedforward networks, convolutional networks, and recurrent networks. Data loaders for standard tabular data, as well as image, text, and sequence data, are provided. It is also possible to train a neural network and use it as a feature extractor to provide suitable input data for another learning algorithm implemented in Weka, such as a support vector machine. Because the neural network predictors in the package are standard Weka "classifier" objects, they can be used and deployed in the same way as other types of predictive models generated by learning algorithms in Weka.

\section{Problems and Background}

Recent years have seen many breakthroughs achieved with deep neural networks, delivering state-of-the-art results in machine learning tasks such as image and document classification. Companies make use of the latest deep learning breakthroughs by benefiting from a quickly evolving software environment comprising different deep learning frameworks. Major frameworks are TensorFlow ${ }^{4}$, PyTorch [2], Deeplearning4j, CNTK [3] and Caffe 4]. Among these frameworks, Deeplearning4j is the most suitable one for integration with Weka [1] because it is also implemented in Java. It supports well-known deep learning architectures such as convolutional neural networks and recurrent neural networks, e.g., long short-term memory networks [5], and training on graphics processing units.

Development of Deeplearning4j has focused on business applications: it is primarily used as an API that enables programmatic integration of deep learning into larger software systems. Our aim with its integration into Weka, which is widely used for research, education and model development through experimentation, is to expand its scope into these areas and provide GUIbased user interaction.

\footnotetext{
${ }^{3}$ https://waikato.github.io/weka-wiki/arff_stable/

${ }^{4}$ https://www.tensorflow.org/
} 


\section{Related Tools}

Software such as Knime [6], Barista [7] and Expresso [8] that exposes deep learning in a GUI use the Python libraries TensorFlow, Keras $5^{5}$ or Caffe as the backend. The user has to manually set up Python using virtual environments or Anaconda ${ }^{6}$ and may have to resolve dependencies of the corresponding deep learning framework. In contrast, our package enables straightforward installation through Weka's GUI by using Java-based components.

\section{Software Framework}

WekaDeeplearning4j is built as a Weka package and thus makes Deeplearning4j models available in the whole Weka environment. The core models available in the package are: 1) D14jMlpClassifier, which enables creation of arbitrarily deep feedforward neural networks, including convolutional neural networks, and 2) RnnSequenceClassifier, which allows training recurrent neural networks on sequential data such as documents. An appealing feature of our tool, described in Section 4.2, is the support for processing two of the most popular data types in deep learning: images and text.

\subsection{Network Configuration}

The configuration of the Dl4jMlpClassifier in the Weka GUI is shown in Figure 1a. The main configuration window exposes a set of basic parameters, such as the number of epochs to train the network, the iterator used to load data (image, textual, tabular or sequence data), an early stopping scheme, and the layer architecture. Fine tuning of configurations can be performed in the network configuration window (not shown here). Through this window it is possible to additionally specify network hyper parameters such as the optimization algorithm, the optimization strategy (updater), regularization factors, initialization methods and more. These configurations are globally applied for each layer that is listed in layer specification.

The layer specification option in the basic model setup window enables the user to create network architectures by stacking different types of neural network layers on top of one another. It is also possible to load a predefined model architecture from the zooModel option, which can then be

\footnotetext{
5 https://github.com/fchollet/keras

6 https://anaconda.com/
} 


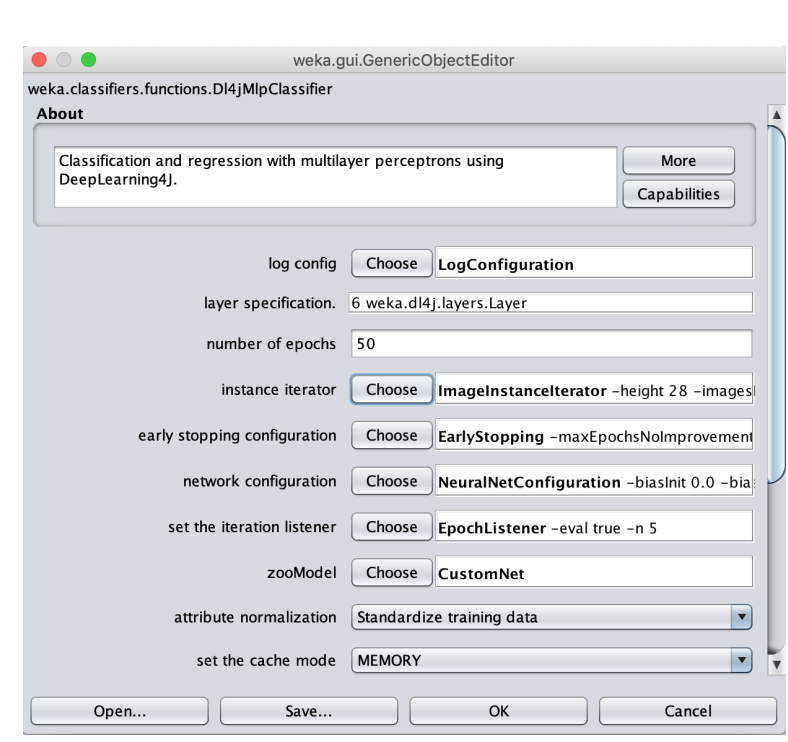

(a)

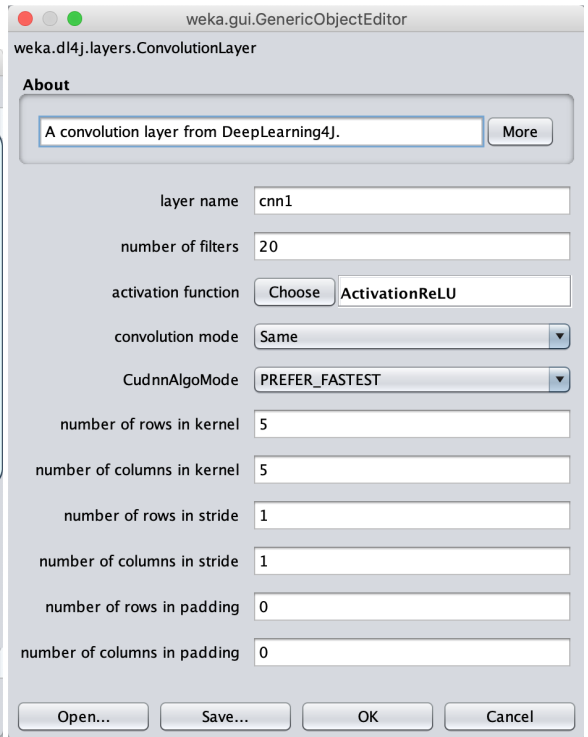

(b)

Figure 1: Configuration of the D14jMlpClassifier model. Figure 1a is the main model configuration window that is shown in the Weka GUI when selecting Dl4jMlpClassifier. From here, further nested configurations can be reached, such as the ConvolutionLayer setup shown in Figure $1 \mathrm{~b}$ and more.

further customized through the layer specification. Each element in the layer list can be chosen from the list of available layers and configured with layer specific options. Figure $1 \mathrm{~b}$ shows the configuration of a ConvolutionLayer, giving options for kernel size, stride, padding and more.

The D14jMlpClassifier can be used for both regression and classification by choosing appropriate loss functions. Additionally, once a classifier has been trained, it is possible to use the serialized D14jMlpClassifier in the D14jMlpFilter preprocessing tool for feature extraction (not shown here).

\subsection{Data Loading}

The package provides so called InstanceIterators to load a given dataset in the correct shape. Each iterator enables setting a batch size that determines the size of the mini batches used for training a network.

\subsubsection{Image Data}

To perform image classification with the package, a dataset must be represented as an ARFF file with one string attribute whose values contain the 
paths of the image files and another attribute with the corresponding target class values. This file can be loaded as the base dataset in the Weka GUI.

To process this data with deep learning, the instance iterator in Figure 1a has to be set to ImageInstanceIterator. This iterator additionally specifies the image base directory, the image height and width, and the number of image channels.

\subsubsection{Text Data}

In deep learning settings, textual data instances (e.g., documents or sentences) are generally represented as sequences of discrete tokens (e.g., words or characters), where each unique token is mapped into a dense vector referred to as an "embedding" [9, 10].

The RnnTextEmbeddingInstanceIterator accepts datasets that contain text data and maps each document into an embedding space, based on word embeddings provided by a lookup table through the location of word vectors option. This iterator can be used with the RnnSequenceClassifier for sequence classification and regression.

Word embeddings can also be generated from the input data with the D14jStringToWord2Vec and D14jStringToGlove filters that implement the Word2Vec [10] and Glove [9] models respectively.

\section{Conclusions}

We have presented an extension to Weka that enables users to create, train, and test deep neural networks using Weka's GUI, employing Deeplearning $4 \mathrm{j}$ as the backend. It is targeted at researchers and data science practitioners who want to experiment with deep learning, opening it up to users who prefer GUI-based interaction and want to minimize opportunity cost associated with the setup of software. The extension also allows the incorporation of deep learning models into users' existing Weka workflows.

From a research perspective, our tool provides a unified environment for comparing deep learning models against other machine learning techniques. It also enables application of deep learning in conjunction with many of the "meta" learning schemes implemented in Weka, e.g., cost-sensitive learning, conditional density estimation, and ordinal classification, which opens up opportunities for new research and applications. 


\section{Acknowledgements}

This work was supported by project 15-UOW-094 of the Marsden Fund of New Zealand. Felipe Bravo-Marquez's work on the text mining aspects of the package was funded by Millennium Institute for Foundational Research on Data.

\section{References}

[1] E. Frank, M. A. Hall, I. H. Witten, The WEKA workbench, Online Appendix for "Data Mining: Practical Machine Learning Tools and Techniques", Morgan Kaufmann, Fourth Edition (2016).

[2] A. Paszke, S. Gross, S. Chintala, G. Chanan, E. Yang, Z. DeVito, Z. Lin, A. Desmaison, L. Antiga, A. Lerer, Automatic differentiation in $\mathrm{Py}-$ Torch, NIPS Autodiff Workshop, Long Beach, CA, USA (2017).

[3] F. Seide, A. Agarwal, CNTK: Microsoft's open-source deep-learning toolkit, in: Proc 22nd Int Conf on Knowledge Discovery and Data Mining, ACM, New York, NY, USA, 2016, p. 2135.

[4] Y. Jia, E. Shelhamer, J. Donahue, S. Karayev, J. Long, R. Girshick, S. Guadarrama, T. Darrell, Caffe: Convolutional architecture for fast feature embedding, in: Proc 22nd Int Conf on Multimedia, ACM, New York, NY, USA, 2014, pp. 675-678.

[5] J. Schmidhuber, Deep learning in neural networks: An overview, Neural networks 61 (2015) 85-117.

[6] M. R. Berthold, N. Cebron, F. Dill, T. R. Gabriel, T. Kötter, T. Meinl, P. Ohl, C. Sieb, K. Thiel, B. Wiswedel, KNIME: The Konstanz Information Miner, in: Data Analysis, Machine Learning and Applications, Springer, 2008, pp. 319-326.

[7] S. Klemm, A. Scherzinger, D. Drees, X. Jiang, Barista - a graphical tool for designing and training deep neural networks (2018). arXiv: 1802.04626 .

[8] J. H. Dholakiya, R. K. Sarvadevabhatla, R. V. Babu, Expresso: A userfriendly GUI for designing, training and using convolutional neural networks (2015). arXiv:1505.06605. 
[9] J. Pennington, R. Socher, C. Manning, Glove: Global vectors for word representation, in: Proc 19th Conf on Empirical Methods in Natural Language Processing, ACL, 2014, pp. 1532-1543.

[10] T. Mikolov, I. Sutskever, K. Chen, G. S. Corrado, J. Dean, Distributed representations of words and phrases and their compositionality, in: Neural Information Processing Systems 26, 2013, pp. 3111-3119. 


\section{Required Metadata}

\begin{tabular}{|c|c|c|}
\hline Nr. & $\begin{array}{l}\text { (executable) Software metadata } \\
\text { description }\end{array}$ & Please fill in this column \\
\hline S1 & Current software version & 1.5 .11 \\
\hline S2 & $\begin{array}{l}\text { Permanent link to executables of } \\
\text { this version }\end{array}$ & $\begin{array}{l}\text { https://github.com/Waikato/ } \\
\text { wekaDeeplearning4j/tree/v1.5. } \\
11\end{array}$ \\
\hline S3 & Legal Software License & GPLv3 \\
\hline $\mathrm{S} 4$ & $\begin{array}{l}\text { Computing platform/Operating } \\
\text { System }\end{array}$ & Linux, OS X, Microsoft Windows \\
\hline S5 & $\begin{array}{l}\text { Installation requirements \& depen- } \\
\text { dencies }\end{array}$ & $\begin{array}{l}\text { Java } 8 \text { or higher, Weka } 3.8 .1 \text { or } \\
\text { higher }\end{array}$ \\
\hline \multirow[t]{2}{*}{ S6 } & \multirow{2}{*}{$\begin{array}{l}\text { If available, link to user manual - if } \\
\text { formally published include a refer- } \\
\text { ence to the publication in the refer- } \\
\text { ence list }\end{array}$} & https://deeplearning.cms. \\
\hline & & waikato.ac.nz/ \\
\hline S7 & Support email for questions & $\begin{array}{l}\text { wekalist@list.scms. waikato. } \\
\text { ac.nz }\end{array}$ \\
\hline
\end{tabular}

Table 1: Software metadata

\begin{tabular}{|c|c|c|}
\hline Nr. & Code metadata description & Please fill in this column \\
\hline $\mathrm{C} 1$ & Current code version & 1.5 .11 \\
\hline \multirow[t]{2}{*}{$\mathrm{C} 2$} & \multirow{2}{*}{$\begin{array}{l}\text { Permanent link to code/repository } \\
\text { used of this code version }\end{array}$} & https://github.com/Waikato/ \\
\hline & & wekaDeeplearning4 $j$ \\
\hline $\mathrm{C} 3$ & Legal Code License & GPLv3 \\
\hline $\mathrm{C} 4$ & Code versioning system used & git \\
\hline C5 & $\begin{array}{l}\text { Software code languages, tools, and } \\
\text { services used }\end{array}$ & Java 8, Gradle, Weka \\
\hline C6 & $\begin{array}{l}\text { Compilation requirements, operat- } \\
\text { ing environments \& dependencies }\end{array}$ & $\begin{array}{l}\text { JDK } 8 \text { or higher, Weka } 3.8 .1 \text { or } \\
\text { higher }\end{array}$ \\
\hline $\mathrm{C} 7$ & $\begin{array}{l}\text { If available Link to developer docu- } \\
\text { mentation/manual }\end{array}$ & $\begin{array}{l}\text { https://waikato.github. } \\
\text { io/wekaDeeplearning4j/ } \\
\text { overview-summary.html }\end{array}$ \\
\hline $\mathrm{C} 8$ & Support email for questions & $\begin{array}{l}\text { wekalist@list.scms.waikato. } \\
\text { ac.nz }\end{array}$ \\
\hline
\end{tabular}

Table 2: Code metadata 